\title{
The Impact of the COVID-19 Pandemic on Intimate Partner Violence Advocates and Agencies
}

\author{
Rebecca Garcia $^{1} \cdot$ Cynterria Henderson $^{2} \cdot$ Kimberly Randell $^{3,4,5} \cdot$ Andrés Villaveces $^{6} \cdot$ Abbey Katz $^{7} \cdot$ Fatimah Abioye $^{8}$. \\ Sarah DeGue ${ }^{6} \cdot$ Kelley Premo $^{2} \cdot$ Summer Miller-Wallfish ${ }^{2} \cdot$ Judy C. Chang ${ }^{9} \cdot$ Elizabeth Miller $^{2} \cdot$ Maya I. Ragavan $^{10,11}$
}

Accepted: 20 October 2021 / Published online: 26 October 2021

(c) The Author(s), under exclusive licence to Springer Science+Business Media, LLC, part of Springer Nature 2021

\begin{abstract}
Relatively few studies have considered the impact of the COVID-19 pandemic on intimate partner violence (IPV) advocates or the agencies where they work. In this study, based on United States IPV advocates' experiences working with survivors during the COVID-19 pandemic, we conducted interviews to explore: 1) personal challenges and resilience working as IPV advocates during the COVID-19 pandemic; 2) how agencies adapted to the pandemic to support IPV survivors and advocates; and 3) specific needs and challenges of culturally-specific agencies. We conducted semi-structured interviews with 53 IPV advocates from June to November 2020. Participants were included if they worked directly with survivors, identified as an IPV advocate, worked at a US-based agency, and spoke and understood English. We created a sampling matrix to ensure adequate representation from IPV advocates serving survivors from communities which have been marginalized. Interviews were conducted through a virtual platform by a trained member of the research team. We used an inductive thematic analysis approach, with weekly coding meetings to resolve discrepancies in coding. Five themes emerged from the data: 1) IPV advocates described how working as an IPV advocate during the COVID-19 pandemic impacted them personally; 2) agencies developed new methods of addressing IPV advocates' needs; 3) agencies developed new solutions to address pandemicrelated client needs; 4) transitioning advocacy work to virtual formats created challenges but also opportunities and; 5) pandemic limitations and impacts compounded pre-pandemic challenges for culturally specific agencies. IPV advocates are frontline workers who have played essential roles in adjusting services to meet survivor needs during the COVID-19 pandemic while simultaneously coping with pandemic impacts on themselves and their agencies. Developing inter-agency collaborations and promoting advocates' safety and wellbeing during future public health crises will help support IPV survivors.
\end{abstract}

Keywords Intimate partner violence $\cdot$ COVID-19 pandemic $\cdot$ Intimate partner violence advocates · Qualitative description; culturally-specific agencies; structural inequities

\section{Introduction}

Intimate partner violence (IPV), defined by the US Centers for Disease Control and Prevention as "physical violence, sexual violence, stalking, or psychological aggression by a current or former partner or spouse," (Breiding et al., 2015) is a pervasive public health problem. One in four women in the United States have experienced physical violence, sexual violence, and/or stalking with IPV related impact; 1 in 3

Rebecca Garcia and Cynterria Henderson are co-first authors

Maya I. Ragavan

ragavanm@chp.edu

Extended author information available on the last page of the article have experienced psychological abuse (Smith et al., 2018). World Health Organization data shows that 1 in 3 women globally have experienced IPV (World Health Organization, 2021). IPV occurs within broader political, structural, and socio-ecological contexts and survivors face unique challenges during natural disasters, pandemics, and times of increased stress. For example, a California-based study found a tripling of IPV-related emergency department visits during and after the Great Recession (2007-2015) compared with the 7 year period before the recession (2000-2007; Medel-Herrero et al., 2020). Similarly, in a sample of postpartum women who lived through Hurricane Katrina, strong associations were found between experiencing damage from the storm and emotional and physical IPV (Harville et al., 2011). 
One such time of increased stress has been the coronavirus 2019 (COVID-19) pandemic, which has created devastating health and economic impacts globally (Bauer et al., 2021). In the early stages of the pandemic, a flurry of commentaries and lay press articles expressed concern about increased IPV frequency and severity due to the multiple interweaving impacts of the pandemic, compounded with widespread physical distancing measures (Campbell, 2020; Evans et al., 2020; Ragavan et al., 2020a; Ragavan et al., 2020b; United Nations, 2021). A recent meta-analysis of studies examining IPV during the COVID-19 pandemic reported that most study estimates were indicative of an increase in IPV during the pandemic (Piquero et al., 2021). Additionally, studies have indicated an increase in IPV severity during the pandemic. As an example, Gosangi et al. (2021) noted a decline in the number of patients seeking healthcare for IPV-related injuries but of those that sought care, the severity of injuries was greater during the pandemic compared with previous years. Similarly, a study by Gresham et al. (2021) noted associations between increased COVID-19 stressors and physical IPV victimization.

In addition to an increase in IPV frequency and severity, IPV survivors have faced challenges during the pandemic. A systematic review published in August 2020 on the impact of the pandemic on IPV survivors reported that survivors faced difficulty meeting basic needs, coercive control by abusive partners, unique safety concerns due to physical distancing, and difficulties accessing resources (Sánchez et al., 2020). Additional studies have affirmed these findings. For example, a study from Chicago showed an increase in calls to the police for issues related to domestic violence but a decrease in arrests when compared to previous years (Bullinger et al., 2021). Studies have also shown that abusive partners have used the health and economic devastation of the COVID19 pandemic to control survivors (Lyons \& Brewer, 2021; Ragavan et al., 2021).

One way to promote the safety and wellness of IPV survivors and their families amidst the health and economic stressors amplified during the pandemic is to bolster support systems. IPV agencies are an important source of support and resources for IPV survivors in the United States. IPV agencies in the United States first emerged in the 1970s and initially focused on providing confidential residential services for women who experienced physical abuse, with agencies often sustained through informal networks and grassroots efforts. Today, IPV agencies provide a variety of essential services such as emergency shelter and transitional housing, children's support and mental health services, transportation assistance, court accompaniment and legal counseling, prevention and educational programming, and therapy and mental health services (Macy et al., 2009; NNEDV, 2020). Currently, there are over 1800 agencies throughout the United States that serve approximately
77,000 survivors and children daily (NNEDV, 2020). Within an IPV agency, direct service work is often conducted by IPV advocates, defined as "anyone who responds directly to help family violence victims in an organizational context" (Davies \& Lyon, 2014). Advocates work within a non-directive service model that centers empowering and supporting survivors to guide their own healing (Sullivan \& Goodman, 2019). Advocates, who can either be paid staff or volunteers, go through extensive training about IPV prior to working with survivors (Davies \& Lyon, 2014; Macy et al., 2009). They perform both individual advocacy with and for IPV survivors as well as systems advocacy which works to decrease institutional barriers for rendering services and resources to survivors (Sullivan \& Goodman, 2019).

IPV advocates and agencies have been on the frontlines during the pandemic, continuing to offer services to meet survivor needs. However, relatively few studies have considered how the COVID-19 pandemic has impacted IPV advocates or the agencies where they work. Research on other frontline workers have demonstrated experiences of burnout during the COVID-19 pandemic. For example, in a US based study, Rodriguez et al. (2020) found the COVID19 pandemic had induced moderate to severe levels of stress at work and home among emergency medicine physicians. A global study of frontline healthcare workers from 60 countries showed that half of 2707 respondents experienced burnout during the pandemic (Morgantini et al., 2020). Additional research has shown how other professionals like law enforcement officers (Stogner et al., 2020), university professors (Van Der Feltz-Cornelis et al., 2020), and veterinarians (Mair et al., 2021) are facing workplace stress due to the COVID-19 pandemic. Despite being frontline workers, less work has considered the experiences of IPV advocates or agencies. Wood et al. (2020) conducted surveys with staff from IPV and sexual assault agencies asking about stress, work, and health and safety planning prior to and during the pandemic. In this study, over $84 \%$ of respondents shared that they experienced an increase in stress during the COVID-19 pandemic. However, apart from this study, to our knowledge little work has considered the ways that IPV advocates or agencies have adapted to the COVID-19 pandemic.

Further, few studies have considered the experiences of culturally-specific IPV agencies, defined as organizations centering the cultural experiences of their clients, which serve as important providers for IPV survivors from communities which have been marginalized (e.g., LGBTQIA+-identifying survivors, immigrants, racial and ethnic minority-identifying survivors; Serrata et al., 2017). Culturally-specific agencies are critical as survivors from communities which have been marginalized may face unique challenges and be cut off from help-seeking resources, disparities driven by structural inequities within the education, healthcare, economic, and legal systems (Bermea et al., 
2019; Grady et al., 2019; Ragavan et al., 2020c; Sokoloff \& Dupont, 2005; Stockman et al., 2015). The work of culturally-specific agencies is particularly important to examine during the COVID-19 pandemic, which has disproportionately impacted Black, Latinx, American Indian/Alaska Native, and LGBTQIA+ identifying peoples, as well as those living in poverty (Azar et al., 2020; Macias Gil et al., 2020; Phillips et al., 2020; Tai et al., 2021). Further, studies have shown that IPV survivors from communities which have been marginalized have faced compounding challenges during the COVID-19 pandemic, rooted in pre-existing inequities (Ragavan et al., 2021; Tahirih Justice Center, 2020).

Understanding the challenges faced by advocates and agencies during the COVID-19 pandemic and identifying how to support these essential service providers can, in turn, help ensure IPV survivors in the United States are being supported and having their needs addressed. Additionally, addressing the challenges faced by IPV advocates and their agencies and leveraging their capacity for resilience can help combat advocate stress and burnout during the COVID-19 pandemic. Such strategies are essential for ensuring the sustainability of the victim services workforce given the severe stressors associated with this pandemic and documented increases in prevalence and severity of IPV. Therefore, in this study, we conducted interviews with US-based IPV advocates to explore: 1) personal challenges and resilience working as IPV advocates during the pandemic; 2) how agencies adapted to support IPV survivors and advocates during the pandemic; and 3) specific needs and challenges of culturally-specific agencies.

\section{Methods}

\section{Study Conceptualization and Design}

This article describes results from a project examining IPV advocates' perspectives on the impact of the COVID-19 pandemic on IPV survivors. Our original intent with this research was to elucidate IPV survivors' experiences and recommendations to support survivors during the pandemic. However, during our first two interviews, advocates also shared how the COVID-19 pandemic had impacted them, as well as how their agencies had to adapt quickly during the pandemic. We recognized these topics, which emerged inductively, as critical to our overall study. Therefore, our team decided to add questions focused on how the COVID19 pandemic impacted IPV advocates and the agencies where they work, results of which are shared in this article. Results on how the COVID-19 pandemic impacted IPV survivors are available elsewhere (Ragavan et al., 2021).

We used a descriptive qualitative approach to frame this study. Qualitative description is "defined as research designed to produce a low-inference description of a phenomenon. (p.13; Kahlke, 2014)" Methodologically, qualitative description often relies on semi-structured interviews as a data collection technique, and, in an effort to stay close to the data, uses an inductive analysis approach, with the majority of codes emerging from the data. We chose this approach because it meets our research aims of describing advocates' experiences during the pandemic and amplifying their voices through an inductive data analysis process (Kahlke, 2014).

\section{Study Team}

Our study team was a multi-disciplinary group representing academic institutions, Futures Without Violence, the American Academy of Pediatrics, and the Centers for Disease Control and Prevention's (CDC) Division of Violence Prevention. This diverse team allowed for representation of various concerns and insights, continuous evaluation of sources of potential bias, and data triangulation from multiple expert and experiential perspectives (Patton, 2015). The University of Pittsburgh Institutional Review Board deemed this study exempt.

\section{Participants}

Study inclusion criteria included: 1) identifying as an IPV advocate; 2) working directly with IPV survivors; 3) working at a US-based agency; and 4) being able to complete the interview in English. For this component of the larger study, participants who did not spend time engaged in direct work with families experiencing IPV (e.g., development or administrative staff) were excluded.

\section{Development of the Interview Guide}

An interview guide was developed by the research team and revised after pilot testing with one IPV advocate. The interview guide was broad and explored advocates' perceptions of the COVID-19 pandemic's impact on IPV, IPV victims, themselves as IPV advocates, their work, and their agencies. Related to this analysis and manuscript, we asked open-ended questions addressing: 1) specific challenges faced by IPV advocates; 2) ways that IPV agencies have adapted in response to the COVID-19 pandemic; and 3 ) emerging or innovative practices IPV agencies have adopted to serve IPV survivors during the COVID-19 pandemic. Interview questions related to results presented in this manuscript are available in Appendix 1. 


\section{Participant Recruitment}

Participant recruitment occurred through announcements on professional listservs and word-of-mouth. Futures Without Violence used their networks, connections, and group distribution lists to ensure information and invitations for study participation were distributed widely across the US and territories. The team identified five groups of historically-marginalized IPV survivors (persons who identify as Black; immigrant and non-English speaking; LGBTQIA+; living with a disability; American Indian/Alaska Native) and intentionally recruited at least one IPV advocate that selfidentified as serving each of those communities. We also recruited IPV advocates from a variety of states, as well as those who worked at culturally specific agencies. Eligible participants emailed or called our study number to set up an interview.

\section{Data Collection}

Trained study team members conducted virtual interviews through a video-conference. Team members reviewed the study objectives, process, and protections and obtained participant's verbal consent. Interviews were audio-recorded, conducted in English, and lasted 45 to $90 \mathrm{~min}$. The interviewer obtained demographic information including race/ ethnicity, gender identity, region of the country, and number of years working at the agency. We also asked advocates whether they worked with IPV survivors from communities which have been marginalized, as described above, as well as whether they worked at a culturally specific IPV agency (self-identified). After completing the interview, participants received a $\$ 30$ electronic gift card. Interviews continued until we reached thematic saturation, or when no new themes emerged, and when the team felt there had been adequate regional diversity and inclusion of advocates from culturally-specific agencies (Guest et al., 2006).

\section{Data Transcription and Analysis}

The research team used an inductive, thematic-analysis approach for coding and analysis, developing codes as they emerged from the data (Braun \& Clarke, 2006; Patton, 2015). We chose an inductive approach to align ourselves with a descriptive qualitative technique and to closely understand the perspectives of advocates during this unprecedented public health emergency. Audio recordings were transcribed verbatim and uploaded into the Dedoose software program (version 7.5.16) to support the organization and management of codes. Two coders independently coded each transcript line by line (Braun \& Clarke, 2006; Dedoose, 2016; Patton, 2015), iteratively developing a codebook containing codes and their definitions. A consensus coder then reviewed the coding and noted any discrepancies. The coding team met weekly to discuss and resolve discrepancies. After coding 25 transcripts, the coding team identified minimal discrepancies; therefore, we shifted to co-coding every third transcript. The full team met weekly to review emerging codes, make iterative changes to the interview guide, and consolidate codes into themes. To further triangulate our emerging data, we held monthly meetings with a group of 25 partners affiliated with national and regional violence prevention and victim services organizations around the United States. Emerging codes and themes were shared with these partners and to help shape the results based on their personal and professional experiences.

\section{Results}

We conducted interviews with 53 IPV advocates working in 25 states in the United States between July 2020 and November 2020. The majority of respondents (92\%) identified as female. Eleven (21\%) advocates described working at culturally specific agencies, with an additional $3(6 \%)$ working with culturally specific programs within a mainstream agency. Advocates serve survivors from a variety of communities which have been marginalized (see Table 1).

Five themes emerged from the data: 1) IPV advocates described how working as an IPV advocate during the COVID-19 pandemic impacted them personally; 2) agencies developed new methods of addressing IPV advocate needs; 3 ) agencies developed new solutions to address pandemic-related client needs; 4) transitioning advocacy work to virtual formats created both challenges and opportunities; and; 5) pandemic limitations and impacts compounded prepandemic challenges for culturally specific agencies.

\section{IPV Advocates Described how Working as an IPV Advocate during the COVID-19 Pandemic Impacted them Personally}

Participants shared the challenges they experienced working as IPV advocates during the COVID-19 pandemic, including mental health symptoms, decreased interaction with other advocates and colleagues, and working from home. Despite these challenges, advocates also described strategies of resilience and perseverance to their profession.

Several advocates described how the pandemic impacted their mental health. An advocate stated: "In the beginning, I was not doing so well. I think everyone that was around me wasn't. Just the uncertainties of everything really took a toll on my mental health." Advocates also noted balancing their mental health with dedication to their work: "Working with survivors that are going through all this fear and everything, and at the same time, the staff is also going through it...I'm 
Table 1 Demographic characteristics of intimate partner violence advocates (total $\mathrm{n}=53$ ) who participated in interviews

\begin{tabular}{ll}
\hline Race/Ethnicity & Participants (n/\%) \\
\hline Asian & $5(6 \%)$ \\
Black/African American & $6(11 \%)$ \\
Latinx & $7(13 \%)$ \\
American Indian/Alaska Native & $4(8 \%)$ \\
White & $30(57 \%)$ \\
Other & $3(6 \%)$ \\
Gender & Participants (n/\%) \\
Female & $49(92 \%)$ \\
Male & $1(2 \%)$ \\
Transgender, Gender queer, Non-binary & $3(6 \%)$ \\
Years as an advocate & \\
Minimum & 5 months \\
Maximum & 46 years \\
Average (years) & 10 years \\
Region & Participants (n/\%) \\
Midwest & $12(22 \%)$ \\
Northeast & $13(25 \%)$ \\
South & $10(19 \%)$ \\
West & $18(34 \%)$ \\
Culturally-specific & Participants (n/\%) \\
Agency & $11(21 \%)$ \\
Program & $3(6 \%)$ \\
Populations Served (Majority) & Participants (n/\%) \\
Black & $11(21 \%)$ \\
Disabled & $2(4 \%)$ \\
Immigrant & $15(28 \%)$ \\
LGBTQIA+ & $8(15 \%)$ \\
Limited English Proficiency & $11(21 \%)$ \\
Indigenous & $8(15 \%)$ \\
\hline &
\end{tabular}

scared because I may get sick but I need to help because this is my job and this is my calling."

Advocates described how connection with peers was paramount to their work and pandemic-related restrictions created collaboration barriers and limited interactions. An advocate said: "You used to be with everyone. You could hear people. You could go to people's office... Now we don't have that. I think right now it's important to continue having those meetings to check up on each other." Another advocate discussed the increased challenge communicating with their colleagues: "I can't just dial my coworker's extension because not everyone is using the same...app that I'm using... It's much harder for me to reach out to people."

Many advocates noted challenges to working from home, a practice many agencies put in place when the pandemic started. An advocate discussed navigating traumatic stories in their homes: "It's really challenging for me because [before the pandemic] I had a routine and I' $m$ physically in a different place. .. I can go into a different room but it's like my clients are here in my home with me." Another advocate shared that her daughter had been hearing stories and experiences of clients:

I picked up [my daughter] at daycare and her babysitter has eczema. She had an outbreak on her neck. My daughter, who doesn't even know her shapes, sat in this woman's lap and said, "Is your boyfriend hitting you?" My three-year-old is now screening people for intimate partner violence in actually a very empathetic and correct type of way. I was super proud of her, but as soon as that happened, I knew that she was hearing me talk about this stuff too much.

Advocates highlighted the necessity of creating boundaries and routines to navigate survivors' interactions from home: "Initially there were advocates who were very opposed to Zoom ${ }^{\circledR}$... because I could tell that they felt like this was blurring a boundary for them... We need to be really boundaried [sic] for this to be sustainable, but the pandemic is forcing us to completely change our boundaries." Another advocate shared their efforts to care for themselves: "I knew my team counted on me so I really tried to take care of myself, getting up early in the morning to try to get a little workout in and still get ready for my work."

Despite the many challenges that advocates faced, they also spoke about the resilience they recognized in themselves, their co-workers, their agencies, and their communities. One advocate highlighted individual-level advocate resilience: "I remember when they announced here, 'Oh, we're gonna [sic] be home three weeks,' everybody was freakin' out, 'Three weeks? This is unbearable, we cannot do it. What will happen to our clients?' Now, it's been five months and they never stopped [working]." Another advocate also spoke of community-level resilience: "[The pandemic] highlights just how resilient our communities always have been. I think it's a reminder, we all have that within us and we have that capacity to just to do more community care and be there for one another."

Advocates also shared how the pandemic bolstered their connections with some of their clients. An advocate noted that adapting to the pandemic and transitioning to remote advocacy developed their ability to provide survivor-centered care: "I think it has made me reflect on my schedule and try to be more available to people outside of typical hours. I think that availability has instilled confidence [in our services] in the people, in some of the service users that I'm working with." Another advocate said: "I miss seeing them. It shows you the strengths and bonds that are made in just helping each other. We learn from the survivors, too. It's coming together and growing and getting through together." 


\section{Agencies Developed New Methods of Addressing IPV Advocate Needs}

Advocates highlighted how their agencies responded to their needs, including accommodating sick time, creating opportunities for social engagement, encouraging scheduling adaptations to allow for self-care, and recognizing advocates' childcare needs.

Advocates stated how helpful it was that their agencies allowed them extra sick time during the pandemic: "They [IPV agency] were really generous with the way that time off happened, and we got an extra bank of sick time that was specifically COVID-related." Another advocate spoke about how co-workers helped when they were sick: "I actually had COVID at the beginning of this, so then I was out for a solid three weeks... Other case managers were also helping carry my workload...It's definitely been sort of like a group effort... to pick up the slack when someone gets sick." In another example, one advocate shared how their agency had to re-evaluate and restructure their organizational style to be more supportive to advocates during the pandemic: "Well, it was a scramble to invent a whole new kind of culture... there are some feelings within our hierarchal system in our agency that our management is sometimes making decisions for the advocates without really understanding or asking the full impact of what those changes are going to look like for us."

Given that many advocates started to work from home during the pandemic, some agencies intentionally created opportunities for socialization and connection. An advocate shared: "We do Zoom coffee in the morning a couple times a week. If we wanna meet with our CEO, there's coffee hours with our CEO. Zoom yoga. There's always something." An advocate who worked with American Indian/Alaska Native communities talked about her agency's collaboration with other organizations working with similar communities to provide support for IPV advocates: "We have met at least once a week on Zoom [with a sister tribe]...That's been super great to be able to see and hear our other advocates in this line of work and be like, "Yeah, okay. Great. You're in the same bubble as we are."

Advocates also shared how agencies were able to help them adapt to the pandemic by understanding and adjusting their schedules and work environments due to competing professional and family responsibilities. Advocates described childcare challenges that their agencies helped them to address: "I am parenting my child alone... She didn't have school. I actually wasn't doing so well in the beginning because I couldn't work a full day of work so I had to go down in hours. My job title, everything stayed the same, but my work hours got reduced." Another advocate noted how the IPV agency allowed the advocates to come into the office when they needed a break from working from home: "My kids are going to school virtually. They're constantly interrupting me while I'm working. We have the option to go into the office... if we want to. It's not required. We can go if we feel like we need a day in the office instead of at home."

\section{Agencies Developed New Solutions to Address Pandemic-Related Client Needs}

Advocates found themselves devising new solutions to address survivor need during the COVID-19 pandemic. Some of these include helping survivors meet basic needs, establishing new partnerships, developing innovative housing solutions, and identifying unique outreach opportunities.

Advocates noted how their agencies prioritized provision of financial assistance and resources for survivors. Although advocates described that most agencies provided some form of financial assistance to their clients pre-pandemic, this need grew exponentially during the COVID-19 pandemic given unprecedented financial challenges and needs. An advocate working with a survivor with a disability discussed how they were able to provide a specific desk chair:

They had to transition doing all of their work... seated and at home... They didn't have options of even really doing much in a common area, so... we were able to provide this chair that would have been very out of their price range... We were actually able to use the funds fairly creatively to address some of the struggles that people are experiencing from COVID.

Another advocate spoke about provision of gift cards to their clients for unrestricted use, a resource that they had not offered previously: "We have these $\$ 200$ Visa cards I can send folks. They can use "em to buy food or clothes or whatever they need, and usually there's never money for food. It's been a huge relief for folks 'cause I think food has gotten really expensive."

The COVID-19 pandemic also prompted IPV agencies to establish new partnerships in an effort to work together in meeting survivors' needs: "I feel like normally, all of our agencies throughout the community, we help each other but we also stay in our lanes. Now... we're all coming together to help each other because there's so much going on right now." The advocate further shared how their agency established relationships with community businesses to facilitate survivors safely accessing services.

Agencies found unique solutions to address housing insecurity. Some agencies partnered with local hotels to put clients into alternative housing: "Our collaboration with hotels has been also something really great... I think that that's been important for us to be able to utilize as some other emergency housing when their shelters are full." An advocate who works with survivors living with disabilities noted similar solutions: "We're partnering with different agencies 
that get COVID-19 monies, we aren't always eligible for those, and partner with them to try to get them motels, even if it's only for three nights." Additionally, an advocate discussed how existing partnerships with local landlords presented another solution to survivor housing insecurity: "I went to the property manager and said, "do you have some apartments that you would rent to me to put our clients in, in a secured, locked building with security cameras.' I rented two apartments right away so that I could start putting clients in."

Many agencies had to adjust communication practices, including texting and email, to address privacy concerns for survivors cohabiting with abusive partners: "When the pandemic first hit, we had only the $24 / 7$ crisis line. We developed a crisis email so as to provide people a little bit more freedom and privacy to reach out and connect with us. We saw an increase in client interaction with that." Agencies also implemented new outreach strategies through social media, community leaders, and essential services to assure the public that services were still available. An advocate described their use of social media:

I believe we've always had a pretty strong social media presence, but we've definitely been strengthening it since COVID because-and especially at the beginning when we were closed, we wanted people to know that we were closed, but we were still available. We still had services to offer, and so social media became the best way to get that word out.

Other advocates shared that they attempted to make their information strategically available in places where they knew survivors would be still going in-person: "[The city] was actually great, and they put up a huge...banner with our number on it, trying to make it visible as cars were driving by and a few flyers around town that the city approved."

\section{Transitioning Advocacy Work to Virtual Formats Created both Challenges and Opportunities}

All participants discussed implementation of virtual service provision, which created unique challenges and opportunities. Many advocates felt that virtual advocacy depersonalized the advocate-survivor relationship and made it challenging to build trust and provide holistic care to IPV survivors. An advocate noted:

Contacting clients over the phone has such a less personal feel. That was a challenge for all of my staff, to try to get people to wanna [sic] engage in services when you can't have the warmth of human connection and empathy, where the person would say, "Gosh, I really like you. I will come back," or "I trust you. I will come back." It seemed like we weren't able to long-term engage people.

Advocates also noted how they could no longer support survivors at court, medical appointments, or other potentially challenging situations

[In-person visits] did offer that emotional support for the survivor, but it also provides a lot of information for me, so that when the person is talking to me later about their medical appointment, I'm actually familiar with what the doctor said, and I can help with clarifying things. .. Those are things that I'm not able to do as easily.

Another advocate noted challenges in supporting clients within the virtual legal system: "We're still living in a virtual world, trying to get in court. If they're not tech-savvy with our judicial system, how are they gonna [sic] be able to do that?"

Advocates noted that transitioning to virtual services provided an array of new privacy and confidentiality considerations: "We've always had really stringent privacy and confidentiality standards. [Now] adding the extra layer of like, we're having to do more over the phone advocacy. We're needing more releases from people, and we're not able to meet with people in person." Virtual services were particularly challenging for IPV survivors cohabiting with their abusive partner. An advocate shared: "It was so challenging because, again so many of them are at home still with their abusers. We have to really assess with all the clients who are interested in participating through Zoom." Several unique strategies were used to maintain privacy, including code words, texting hotlines, and password protection or other security features: "We have a code that if someone says [code redacted] that means everyone has to hang up from the [virtual support] group... and we restart it again later."

Despite the many challenges associated with virtual support and services, the shift also provided some benefits. Virtual services were more accessible: "The plus side of the phone appointments is they're-you don't have the transportation hindrance, and it seems like you have a lot more access to it." Similarly, another advocate noted markedly improved attendance when discussing virtual support groups: 'Interestingly, running virtual support groups, I'm getting far better attendance than I was in person...Now, virtually, there's a great need and people are showing up for groups. I'm no longer sitting in community libraries, but we're trying to create a virtual community." Another advocate explained that the transition to virtual services during the pandemic will continue into the future:

Going virtual and using the technology to our advantage right now has been a change which will obviously continue because the virtual presence and the technol- 
ogy is only gonna [sic] get more advanced and more complex as we go. We'll still do in-person presentations, but it's not gonna [sic] be like it was. This has probably jump-started our ability to be able to do that."

\section{Pandemic Limitations and Impacts Compounded Pre-Pandemic Challenges for Culturally Specific Agencies}

Advocates from culturally specific agencies shared many comparable experiences with their counterparts but, notably, these advocates also described an existing lack of resources and capacity that predated the pandemic. Specifically, one advocate noted that adults living with disabilities often have trouble getting their needs met by external service providers: "Nobody, I hate to say it, wants to deal with [adults living with disabilities] because you do have to spend more time. They don't have that time, so they send 'em over to us [even though] they know that we can't [help] them in certain areas." An advocate working with rural American Indian/ Alaska Native communities shared how agency-level financial challenges restrict the resources available to survivors, "There's no funding to create new resources, it's just been the same limited resources."

Advocates from culturally specific agencies also discussed a number of staffing constraints during the pandemic. An advocate who works with survivors identifying as Black and LGBTQIA+ described issues created by expectations that their agency would provide trainings to outside organizations:

We have five people on the team and so we're constantly stretched. The thing that's really incredible is that we're the oldest organization [LGBTQIA+] organization and... there's an expectation to provide the [diversity and inclusion] trainings and be on the panels and do certain things that are difficult to think about. I would say just capacity. Capacity is a big thing.

Similarly, an advocate who works with an immigrant community described challenges keeping up with survivors' legal needs during this time due to staffing limitations: "We've always been limited. We have two, three attorneys. At this point, we only have one. That was another thing that-internally, we were dealing with that, we had just that, but then the referrals went to the sky, was really high."

Additionally, advocates from culturally specific agencies that provide specialized services to non-English speaking populations highlighted long-standing language accessibility barriers which worsened during the pandemic. An advocate who works with South Asian survivors discussed the challenges their clients encountered when applying for government assistance after facing unemployment related to the pandemic.
It was very difficult in applying for anything because most of the information was in English, and for limited English proficiency survivors, that was really hard to understand. A lot of our work was the need to find [and] translate... the forms... It was difficult and there were delays around that.

Similarly, another advocate who works with immigrant and refugee survivors noted: “...[the] people that I work with are monolingual Arabic speaking, and in my agency, most clients are actually not English speakers. We all were supporting clients with those services, just giving them language access and advocating for them."

Culturally specific agencies highlighted unique solutions to serve IPV survivors from communities which have been marginalized. An advocate who works with survivors identifying as Black, rural, and LGBTQIA+ described the implementation of preventative outreach strategies to support survivors during the pandemic: "We wanted to reach out before there might be some fallback... We'll just be like, "Hey. We're here. We believe in you. You've got this. If you need anything, we're here." In response to the language access barriers, an advocate noted, "[Something] that we had done earlier on was translating a lot of the CDC material into various South Asian languages that they posted online. We send [it] to people who spoke that particular language."

\section{Discussion}

To our knowledge, this is one of the first studies examining the impact of the COVID-19 pandemic on IPV advocates and agencies. We found that participants relayed an array of stressors during the pandemic, but also highlighted individual and community-level resilience. Agencies used a variety of strategies to support advocates and serve survivors, including mobilizing a shift to virtual services. Our findings are aligned with a study by Wood et al. (2020) who similarly found that IPV agencies transitioned to virtual services and reported that IPV advocates stress level increased during the pandemic. Our work adds the focus on the needs and experiences of culturally-specific agencies. Like all IPV agencies, culturally-specific agencies face challenges associated with a lack of resources, high work demand, stress, and vicarious trauma (Cummings et al., 2018; Kulkarni et al., 2013; Wood et al., 2019). For culturally specific agencies, however, these challenges are compounded due to deeply entrenched power inequities and interwoven oppressions which must be dismantled (Starr, 2018; USDOJ, 2017). Our study demonstrates that, in addition to their victim advocacy duties, culturally specific agencies are charged with the responsibility of advocating for the needs of survivors from communities which have been marginalized with little support 
and diminished capacity due to limited staffing and funding limitations. This burden is consistent with previous research within academic settings demonstrating a "minority tax", or the burden of additional, uncompensated responsibilities placed on students, faculty, and staff from underrepresented communities (Rodríguez et al., 2015). The ramifications of the minority tax have been well documented; faculty and staff often feel pressured to participate in diversity and inclusion efforts, limiting time spent performing their assigned duties and contributing to exhaustion and burn out (Campbell and Rodríguez, 2019; Rodríguez et al., 2015). Although the minority tax has been studied in academia and the medical field, there is little literature on its impact in community-based settings such as IPV agencies. This tax may have worsened during the pandemic, as culturally specific agencies are facing even more direct services responsibility while being asked to stretch to support other agencies.

Participants noted experiencing considerable mental health symptoms working as essential workers during the COVID-19 pandemic. Experiencing vicarious trauma (i.e., the result of exposure and empathic listening to stories of trauma, suffering and violence; Sexual Violence Research Initiative, 2015) is well-documented among IPV advocates and other trauma service providers. During the COVID-19 pandemic, literature has also described the vicarious trauma healthcare providers are facing (Esterwood \& Saeed, 2020; Santarone et al., 2020). Our study builds on past work to demonstrate how IPV advocates are also experiencing vicarious trauma as they support survivors during the pandemic. Further, many IPV advocates may have experienced abuse themselves thus potentially compounding advocates' mental health symptoms but also allowing advocates to forge connections with IPV survivors which may reflect the resilience noted by advocates during the pandemic (Bemiller \& Williams, 2011; Wood, 2017).

IPV advocates faced challenges bringing trauma work into their home. Discussing trauma on the phone or through virtual platforms with survivors while working at home may potentially expand experiences of vicarious trauma beyond the advocate to their family, including children who may be more frequently at home due to virtual schooling and childcare limitations. These findings align with past research demonstrating that emergency medical services and trauma therapists may experience vicarious trauma working via phone or online technology (Dunkley and Wheland, 2006; Meischke et al., 2018; van Dernoon Lipinsky \& Burk, 2009). Our study demonstrates additional challenges for advocates conducting trauma work from home, and these findings affirm the need to provide emotional support and mental health services during global pandemics or national crises (Fish \& Mittal, 2020).

Our study highlights the emergence of both challenges and unique opportunities with the transition to virtual advocacy. Multiple past studies have noted that use of telemedicine may increase patients' access to care (Brova et al., 2018; Dorsey et al., 2018; Ronis et al., 2017). Our findings align with this work, as advocates described improved accessibility for survivors who may experience difficulties due to transportation or inequitable service design. Conversely, participants in our study noted how virtual services may feel depersonalized, which has been reported by patients and healthcare providers using telemedicine (Sabina Sousa et al., 2020; Kwan et al., 2019; Rohland et al., 2000). Study participants also identified confidentiality and privacy barriers associated with the provision of virtual services for IPV survivors, similar to previous work demonstrating that virtual service provision for LGBTQIA+ youth may be challenging due to lack of private space (Silliman Cohen and Bosk, 2020). IPV survivors may face unique privacy challenges, as their abusive partner may be intentionally eavesdropping on conversations or monitoring technology use, a form of abuse that is well-documented in the literature (Burke et al., 2011; Freed et al., 2017; Woodlock, 2017).

Due to the complex nature of IPV, it is not uncommon for agencies to collaborate with law enforcement, social service personnel, and healthcare workers to ensure that all survivor needs are met (Langenderfer-Magruder et al., 2019; Shorey et al., 2014). Our study builds on past work describing the value of collaborative relationships between IPV agencies and community partners during natural disasters (First et al., 2017). Jenkins and Phillips (2009) noted the challenges New Orleans IPV agencies faced following Hurricane Katrina, when local police, attorneys, and healthcare systems were often overwhelmed and under-resourced, operating within inadequate infrastructure in a post-disaster environment. To address these challenges, our research highlights that developing these collaborations (as part of emergency preparedness) can help meet survivor needs when the survivor's usual social safety net has been depleted through a catastrophic event such as a pandemic. Further, our findings suggest that it is possible to sustain and grow these relationships during a time of crisis.

Many advocates spoke about the pervasiveness of housing insecurity due to the widespread job loss and economic downturn resulting from the COVID-19 pandemic. Past literature describes how IPV survivors are more likely than those who have not experienced IPV to face housing instability and homelessness (Klein et al., 2021; Pavao et al., 2007). The COVID-19 pandemic has exacerbated pre-existing housing insecurity. Space in confidential emergency shelters, which was limited pre-pandemic, has become sparser as agencies restrict availability to implement COVID-19 prevention measures. Further, pandemic prevention measures such as these may compound feelings of restriction that survivors may experience in residential shelter settings. Shelter rules, although well-intentioned, 
can be barriers to trauma-informed care, with consequences as severe as loss of service provision if rules are not followed (Glenn \& Goodman, 2015; Kulkarni et al., 2019; Wood et al., 2020b). Our findings suggest alternative housing options (e.g., hotels) may, in certain circumstances, be a feasible and potentially effective solution even after the pandemic, allowing for a more survivorcentered approach, but requiring a higher level of financial resources to sustain.

We recognize several limitations to this study. We focus on IPV advocates at local IPV agencies and those conducting direct service work. Additional research is needed to describe the role of policies guiding allocation of resources and practices including perspectives of IPV administrators at regional and national agencies. Although our sample included IPV advocates from agencies in diverse regions of the US, we recognize that our recruitment strategy may not have included IPV advocates at smaller or hard-to-reach agencies. We recognize the results of this study come from 53 advocates and may not be generalizable; however, the goal of this qualitative study was to understand heterogeneous experiences in the context of a pandemic.

\section{Implications}

Research Implications Our study sets the stage for future research. The work focuses on US-based advocates; so further research is necessary to understand how the COVID-19 pandemic has impacted IPV agencies globally. More work should focus on the experiences of advocates during disasters and how their experiences can inform disaster planning. Evaluation of some of the innovative practices adopted by agencies during the COVID-19 pandemic is needed to determine how to sustain promising practices. Longitudinal studies, examining the impact of the pandemic on IPV advocates and agencies over time and throughout the post-pandemic recovery period, are also needed. Finally, this study focused on advocates and future research should consider the perspectives of regional and state-level IPV coalitions.

\section{Practice Implications to Support the Health and Wellbeing} of IPV Advocates Multiple strategies also emerged to support IPV advocates, particularly during pandemics or other times when they have to do their work isolated from their colleagues. Providing flexible schedules, childcare, and connection with other advocates may be first steps toward promoting advocate self-care. Additionally, providing confidential and free mental health and peer support services for IPV advocates (such as a peer-to-peer advocate helpline) may also be useful. Sustaining these strategies beyond the pandemic may help support this life-saving work and reduce burnout among IPV advocates.
Practice Implications for Providers and Agencies Supporting IPV Survivors The results of this study highlight multiple recommendations that can be implemented by service providers and agencies supporting IPV survivors, including law enforcement, medical professionals, legal systems, and victim services agencies. Leveraging technology to provide virtual IPV services both when in-person services are not available and for survivors with disabilities will help improve accessibility. Using texting and chat lines, providing easy exit strategies during virtual strategies, and providing services to easily sign consent forms can protect privacy and confidentiality when using technology for IPV survivors. Additionally, it is particularly helpful for service providers to develop collaborative relationships so they can work together to provide survivor-centered care. While such collaborations are especially critical during pandemics or other disasters, building them before pandemics will help to ensure that communication and trust are well established.

Policy Implications for Governmental Agencies Our results demonstrate that IPV advocates are essential components of our healthcare infrastructure and, as such, are essential workers. During the COVID-19 pandemic, allocation of personal protective equipment to IPV advocates and agencies is important to protect advocates while allowing them to continue to address survivors' needs. Additionally, IPV advocates and other front-line providers working with IPV survivors can be considered healthcare workers in terms of vaccine prioritization. This study also shows how incorporating IPV as part of strategic planning and policymaking for public health and emergency preparedness planning can facilitate improved support of IPV survivors. These results also demonstrate the role that policy may play in addressing underlying structural oppressions that may create compounding challenges for IPV survivors as well as elevating policies and practices that address meeting basic needs.

Supporting Culturally Specific Agencies Culturally specific IPV programs and agencies play an important role in supporting survivors from communities which have been marginalized, but they are limited by power differentials and lack of resources (Starr, 2018), both rooted in deep-seated, systemic inequities which were compounded by the COVID19 pandemic. Amplifying the voices of survivors by prioritizing the work of culturally specific agencies is needed, as is building meaningful partnerships with culturally-specific agencies. We also must support culturally specific agencies with capacity building to combat the minority tax many agencies face. A first step toward dismantling power inequities and supporting the work of culturally-specific agencies would be for state and local governments to consider adequately compensating employees and fully funding the agencies that provide these necessary services. Please see 
papers by White Starr for further discussion (Starr, 2018, 2020).

\section{Conclusions}

In this study, we interviewed IPV advocates to learn about the way the COVID-19 pandemic impacted them and the agencies where they work. The pandemic created multiple personal challenges but also opportunities for self and community-care. Agencies adopted new strategies to support survivors and advocates, including shifting to virtual service provision. Culturally-specific agencies have faced unique challenges. Results describe how IPV advocates have been front-line workers during the pandemic providing critical resources to IPV survivors, thus setting the stage for policy and practice changes to ensure that IPV advocates and agencies thrive during the COVID-19 pandemic and in the post-pandemic recovery.

\section{Appendix 1}

Sample Questions from the IPV Advocate interviews:

Please describe the needs you have seen when working with IPV survivors during COVID-19.

Please describe how it has been for you working as an advocate during the pandemic? What changes have you had to make?

Next, I would like to discuss challenges your agency has faced during the pandemic.

Prompt: Has your organization had to change how you reach out to survivors?

Prompt: How has your job or role changed in response to COVID-19?

How has your agency responded to your needs during the pandemic?

How has your agency worked to ensure privacy and confidentiality during virtual meetings and appointments?

Please describe what you need to support your work as an advocate during this pandemic?

Have there been any changes your agency made during the pandemic which you would like to sustain?

For culturally-specific agencies: Please tell us about any specific challenges your or your agency have faced?

Acknowledgements We acknowledge Lauren Risser, Phoebe Balascio, and Janine Talis for their help with data collection and analysis.

Funding This study was funded by a cooperative agreement from the Centers for Disease Control and Prevention (NU38OT000282). Dr. Ragavan is supposed by a National Center for Advancing Translational Sciences University of Pittsburgh KL2 (TR001856; Rubio). Dr. Randell is supported by a National Institute of Child Health and Development K23 (K23HD098299).

The findings and conclusions in this manuscript are those of the author(s) and do not necessarily represent the official position of the Centers for Disease Control and Prevention or the American Academy of Pediatrics.

\section{Declarations}

Conflict of Interest The authors do not have any conflicts of interest to disclose. The findings and conclusions in this manuscript are those of the author(s) and do not necessarily represent the official position of the Centers for Disease Control and Prevention.

\section{References}

Azar, K., Shen, Z., Romanelli, R. J., Lockhart, S. H., Smits, K., Robinson, S., Brown, S., \& Pressman, A. R. (2020). Disparities in outcomes among COVID-19 patients in a large health care system in California. Health affairs (Project Hope), 39(7), 1253-1262. https://doi.org/10.1377/hlthaff.2020.00598

Bauer, L., Broady, K., Edelberg, W., \& O'Donnell, J. (2021, February 02). Ten facts about COVID-19 and the U.S. economy. Retrieved March 17, 2021, from https://www.brookings.edu/research/tenfacts-about-covid-19-and-the-u-s-economy/

Bermea, A. M., van Eeden-Moorefield, B., \& Khaw, L. (2019). Serving queer survivors of intimate partner violence through diversity, inclusion, and social justice. Journal of Gay \& Lesbian Social Services, 31(4), 521-545. https://doi.org/10.1080/10538720.2019. 1653805

Bemiller, M., \& Williams, L. S. (2011). The role of adaptation in advocate burnout: A case of good soldiering. Violence Against Women, 17, 89-110.

Braun, V., \& Clarke, V. (2006). Using thematic analysis in psychology. Qualitative Research in Psychology, 3(2), 77-101. https://doi.org/ 10.1191/1478088706qp063oa

Breiding, M.J., Basile, K.C., Smith, S.G., Black, M.C., Mahendra, R. Intimate partner violence surveillance uniform definitions and recommended data elements. Centers for Disease Control and Prevention, National Center for Injury Prevention and Control. Atlanta, GA; 2015. Retrieved from: https://www.cdc.gov/viole nceprevention/pdf/ipv/intimatepartnerviolence.pdfAccessed $7 / 1 / 2021$

Brova, M., Boggs, K. M., Zachrison, K. S., Freid, R. D., Sullivan, A. F., Espinola, J. A., Boyle,T. P., \& Camargo, C. A. (2018). Pediatric telemedicine use in United States emergency departments. Academic Emergency Medicine, 25(12), 1427-1432. https://doi. org/10.1111/acem.13629

Bullinger, L. R., Carr, J. B., \& Packham, A. (2021). COVID-19 and crime: Effects of stay-at-home orders on domestic violence. American Journal of Health Economics, 7(3), 249-280.

Burke, S. C., Wallen, M., Vail-Smith, K., \& Knox, D. (2011). Using technology to control intimate partners: An exploratory study of college undergraduates. Computers in Human Behavior, 27(3), 1162-1167.

Campbell, A. M. (2020). An increasing risk of family violence during the COVID-19 pandemic: Strengthening community collaborations to save lives. Forensic Science International Reports, 2, 100089.

Campbell, K. M., \& Rodríguez, J. E. (2019). Addressing the minority tax: Perspectives from two diversity leaders on building minority faculty success in academic medicine. Academic Medicine : 
Journal of the Association of American Medical Colleges, 94(12), 1854-1857. https://doi.org/10.1097/ACM.0000000000002839

United Nations. (2021). COVID-19: A gender Lens | UNFPA - United Nations Population Fund. (n.d.). Retrieved January 20, 2021, from https://www.unfpa.org/resources/covid-19-gender-lensAccessed 7/1/2021

Cummings, C., Singer, J., Hisaka, R., \& Benuto, L. T. (2018). Compassion satisfaction to combat work-related burnout, vicarious trauma, and secondary traumatic stress. Journal of interpersonal violence, 886260518799502. https://doi.org/10.1177/ 0886260518799502

Davies, J., \& Lyon, E. (2014). Safety planning with battered women: Complex lives/difficult choices (2nd ed.). Sage.

Dedoose. (2016). Web application for managing, analyzing, and presenting qualitative and mixed method research data (7.5.16). Computer software, Los Angeles, CA: SocioCultural Research Consultants, LLC.

Dorsey, E. R., Glidden, A. M., Holloway, M. R., Birbeck, G. L., \& Schwamm, L. H. (2018). Teleneurology and mobile technologies: The future of neurological care. Nature reviews. Neurology, 14(5), 285-297. https://doi.org/10.1038/nrneurol.2018.31.

Dunkley, J. \& Wheland, T.A. (2006). Vicarious traumatisation in telephone counsellors: Internal and external influences. British Journal of Guidance \& Counselling, 34(4).

Esterwood, E., \& Saeed, S. A. (2020). Past epidemics, natural disasters, COVID19, and mental health: Learning from history as we Deal with the present and prepare for the future. The Psychiatric Quarterly, 91(4), 1121-1133. https://doi.org/10.1007/ s11126-020-09808-4

Evans, M. L., Lindauer, M., \& Farrell, M. E. (2020). A pandemic within a pandemic - intimate partner violence during Covid-19. The New England Journal of Medicine, 383(24), 2302-2304. https://doi.org/10.1056/NEJMp2024046

First, J. M., First, N. L., \& Houston, J. B. (2017). Intimate partner violence and disasters: A framework for empowering women experiencing violence disaster settings. Affilia, 32(3), 390-403. https://doi.org/10.1177/0886109917706338

Fish, J. N., \& Mittal, M. (2020). Mental health providers during covid-19. Public Health Reports, 136(1), 14-17. https://doi.org/ 10.1177/0033354920965266

Freed, D., Palmer, J., Minchala, D. E., Levy, K., Ristenpart, T., \& Dell, N. (2017). Digital technologies and intimate partner violence. Proceedings of the ACM on human-computer interaction, 1(CSCW), 1-22. https://doi.org/10.1145/3134681

Glenn, C., \& Goodman, L. (2015). Living with and within the rules of domestic violence shelters: A qualitative exploration of residents' experiences. Violence Against Women, 21(12), $1481-1506$.

Gresham, A. M., Peters, B. J., Karantzas, G., Cameron, L. D., \& Simpson, J. A. (2021). Examining associations between COVID-19 stressors, intimate partner violence, health, and health behaviors. Journal of Social and Personal Relationships, 38(8), 2291-2307.

Gosangi, B., Park, H., Thomas, R., Gujrathi, R., Bay, C. P., Raja, A. S., et al. (2021). Exacerbation of physical intimate partner violence during COVID-19 pandemic. Radiology, 298(1), E38-E45.

Grady, G., Hinshaw-Fuselier, S., \& Friar, N. (2019). Expanding perspectives: A social inequities lens on intimate partner violence, reproductive justice, and infant mental health. Infant Mental Health Journal, 40(5), 624-639. https://doi.org/10.1002/imhj. 21809

Guest, G., Bunce, A., \& Johnson, L. (2006). How many interviews are enough?: An experiment with data saturation and variability. Field Methods, 18(1), 59-82. https://doi.org/10.1177/15258 22X05279903
Harville, E. W., Taylor, C. A., Tesfai, H., Xu, X., \& Buekens, P. (2011). Experience of hurricane Katrina and reported intimate partner violence. Journal of Interpersonal Violence, 26(4), 833-845. https://doi.org/10.1177/0886260510365861

Jenkins, P., \& Phillips, B. (2009). Battered women, catastrophe, and the context of safety after hurricane Katrina. NWSA Journal., 20(3), $49-68$.

Kahlke, R. M. (2014). Generic qualitative approaches: Pitfalls and benefits of methodological mixology. International Journal of Qualitative Methods, 13(1), 37-52. https://doi.org/10.1177/16094 0691401300119

Klein, L. B., Chesworth, B. R., Howland-Myers, J. R., Rizo, C. F., \& Macy, R. J. (2021). Housing interventions for intimate partner violence survivors: A systematic review. Trauma, Violence \& Abuse, 22(2), 249-264. https://doi.org/10.1177/1524838019836284

Kulkarni, S., Bell, H., Hartman, J. L., \& Herman-Smith, R. L. (2013). Exploring individual and organizational factors contributing to compassion satisfaction, secondary traumatic stress, and burnout in domestic violence service providers. Journal of the Society for Social Work and Research, 4(2), 114-130.

Kulkarni, S. J., Stylianou, A. M., \& Wood, L. (2019). Successful rules reduction implementation process in domestic violence shelters: From vision to practice. Social Work, 64(2), 147-156. https://doi. org/10.1093/sw/swz010

Kwan, J. Y. Y., Croke, J., Panzarella, T., Ubhi, K., Fyles, A., Koch, A., Dinniwell, R., Levin, W., McCready, D., Chung, C., Liu, F., \& Bender, J. L. (2019). Personalizing post-treatment cancer care: A cross-sectional survey of the needs and preferences of well survivors of breast cancer. Current oncology (Toronto, Ont.), 26(2), e138-e146. https://doi.org/10.3747/co.26.4131.

Langenderfer-Magruder, L., Olson, C., Wilke, D. J., \& Alven, L. (2019). RISE up: Facilitating frontline responder collaboration on co-occurring child welfare and intimate partner violence cases. Journal of Interpersonal Violence, 886260519832921. https://doi. org/10.1177/0886260519832921

Lyons, M., \& Brewer, G. (2021). Experiences of intimate partner violence during lockdown and the COVID-19 pandemic. Journal of family violence, 1-9. Advance online publication. https://doi.org/ 10.1007/s10896-021-00260-x.

Macias Gil, R., Marcelin, J. R., Zuniga-Blanco, B., Marquez, C., Mathew, T., \& Piggott, D. A. (2020). COVID-19 pandemic: Disparate health impact on the Hispanic/Latinx population in the United States. The Journal of Infectious Diseases, 222(10), 1592-1595. https://doi.org/10.1093/infdis/jiaa474

Macy, R. J., Giattina, M., Sangster, T. H., Crosby, C., \& Montijo, N. J. (2009). Domestic violence and sexual assault services: Inside the black box. Aggression and Violent Behavior, 14(5), 359-373. https://doi.org/10.1016/j.avb.2009.06.002

Mair, T. S., Mountford, D. R., Radley, R., Lockett, E., \& Parkin, T. D. (2021). Mental wellbeing of equine veterinary surgeons, veterinary nurses and veterinary students during the COVID-19 pandemic. Equine Veterinary Education, 33(1), 15-23.

Medel-Herrero, A., Shumway, M., Smiley-Jewell, S., Bonomi, A., \& Reidy, D. (2020). The impact of the great recession on California domestic violence events, and related hospitalizations and emergency service visits. Preventive Medicine, 139, 106186. https:// doi.org/10.1016/j.ypmed.2020.106186

Meischke, H., Lilly, M., Beaton, R., Calhoun, R., Tu, A., Stangenes, S., Painter, I., Revere, D., \& Baseman, J. (2018). Protocol: A multilevel intervention program to reduce stress in 9-1-1 telecommunicators. BMC Public Health, 18(1), 570. https://doi.org/10.1186/ s12889-018-5471-0

Morgantini, L. A., Naha, U., Wang, H., Francavilla, S., Acar, Ö., Flores, J. M., Crivellaro, S., Moreira, D., Abern, M., Eklund, M., Vigneswaran, H. T., \& Weine, S. M. (2020). Factors contributing to healthcare professional burnout during the COVID-19 pandemic: 
A rapid turnaround global survey. PLoS One, 15(9), e0238217. https://doi.org/10.1371/journal.pone.0238217

National Network to End Domestic Violence (NNEDV). (2020, March 10). 14th Annual Domestic Violence Counts Report. Retrieved March 17, 2021, from https://nnedv.org/wp-content/uploads/2020/ 03/Library_Census-2019_Report_web.pdfAccessed 7/1/2021

Patton MQ. Qualitative Research \& Evaluation Methods. $4^{\text {th }}$ edition. Thousand Oaks, CA; SAGE Publications, 2015.

Pavao, J., Alvarez, J., Baumrind, N., Induni, M., \& Kimerling, R. (2007). Intimate partner violence and housing instability. American Journal of Preventive Medicine, 32(2), 143-146. https://doi. org/10.1016/j.amepre.2006.10.008

Phillips, G., Felt, D., Ruprecht, M. M., Wang, X., Xu, J., Perez-Bill, E., Bagnarol, R. M., Roth, J., Curry, C. W., \& Breach, L. B. (2020). Addressing the disproportionate impacts of the COVID-19 pandemic on sexual and gender minority populations in the United States: Actions towards equity. LGBT Health, 7(6). https://doi.org/ 10.1089/lgbt.2020.0187

Piquero, A. R., Jennings, W. G., Jemison, E., Kaukinen, C., \& Knual, F. M. (2021). Domestic violence during the COVID-19 pandemic: Evidence from a systematic review and meta-analysis. Journal of Criminal Justice, 74, 101806.

Ragavan, M. I., Thomas, K. A., Fulambarker, A., Zaricor, J., Goodman, L. A., \& Bair-Merritt, M. H. (2020a). Exploring the needs and lived experiences of racial and ethnic minority domestic violence survivors through community-based participatory research: A systematic review. Trauma, Violence, \& Abuse, 21(5), 946-963.

Ragavan, M. I., Garcia, R., Berger, R. P., \& Miller, E. (2020b). Supporting intimate partner violence survivors and their children during the COVID-19 pandemic. Pediatrics, 146(3).

Ragavan, M. I., Culyba, A. J., Muhammad, F. L., \& Miller, E. (2020c). Supporting adolescents and young adults exposed to or experiencing violence during the COVID-19 pandemic. The Journal of Adolescent Health, 67(1), 18-20. https://doi.org/10.1016/j.jadoh ealth.2020.04.01

Ragavan, M. I., Risser, L., Duplessis, V., DeGue, S. Villaveces, A., Hurley, T., .... Randell, K. A. (2021). The impact of the COVID19 pandemic on the needs and lived experiences of intimate partner violence survivors in the United States: Advocate perspectives. Violence Against Women. In-press.

Rodríguez, J. E., Campbell, K. M., \& Pololi, L. H. (2015). Addressing disparities in academic medicine: What of the minority tax? BMC Medical Education, 15(1), 6.

Rodriguez, R. M., Medak, A. J., Baumann, B. M., Lim, S., Chinnock, B., Frazier, R., \& Cooper, R. J. (2020). Academic emergency medicine Physicians' anxiety levels, stressors, and potential stress mitigation measures during the acceleration phase of the COVID19 pandemic. Academic Emergency Medicine : Official Journal of the Society for Academic Emergency Medicine, 27(8), 700-707. https://doi.org/10.1111/acem.14065

Rohland, B. M., Saleh, S. S., Rohrer, J. E., \& Romitti, P. A. (2000). Acceptability of telepsychiatry to a rural population. Psychiatric Services, 51(5), 672-674. https://doi.org/10.1176/appi.ps.51.5. 672

Ronis, S. D., McConnochie, K. M., Wang, H., \& Wood, N. E. (2017). Urban telemedicine enables equity in access to acute illness care. Telemedicine Journal and E-Health, 23(2), 105-112. https://doi. org/10.1089/tmj.2016.0098

Sabina Sousa, C., Trigueiro Barbosa, M., Aguiar, R., Benito-Garcia, F., \& Morais-Almeida, M. (2020). What do asthmatic patients think about telemedicine visits? European Annals of Allergy and Clinical Immunology. 10.23822/EurAnnACI.1764-1489.182

Sánchez, O. R., Vale, D. B., Rodrigues, L., \& Surita, F. G. (2020). Violence against women during the COVID-19 pandemic: An integrative review. International Journal of Gynaecology and Obstetrics, 151(2), 180-187. https://doi.org/10.1002/ijgo.13365
Santarone, K., McKenney, M., \& Elkbuli, A. (2020). Preserving mental health and resilience in frontline healthcare workers during COVID-19. The American Journal of Emergency Medicine, 38(7), 1530-1531. https://doi.org/10.1016/j.ajem.2020.04.030

Serrata, J. V., Macias, R. L., Rosales, A., Hernandez-Martinez, M., Rodriguez, R., \& Perilla, J. L. (2017). Expanding evidence-based practice models for domestic violence initiatives: A communitycentered approach. Psychology of Violence, 7(1), 158-165. https:// doi.org/10.1037/vio0000051

Sexual Violence Research Initative. Guidelines for the prevention and management of vicarious trauma among researchers of sexual and intimate partner violence. (2015). Sexual violence research initiative. South Africa.

Shorey, R. C., Tirone, V., \& Stuart, G. L. (2014). Coordinated community response components for victims of intimate partner violence: A review of the literature. Aggression and Violent Behavior, 19(4), 363-371. https://doi.org/10.1016/j.avb.2014.06.001

Silliman Cohen, R. I., \& Bosk, E. A. (2020). Vulnerable youth and the COVID-19 pandemic. Pediatrics, 146(1), e20201306. https://doi. org/10.1542/peds.2020-1306

Smith, S. G., Zhang, X., Basile, K. C., Merrick, M. T., Wang, J., Kresnow, M., \& Chen, J. (2018). The National Intimate Partner and sexual violence survey (NISVS): 2015 data brief - Updated release. National Center for Injury Prevention and Control, Centers for Disease Control and Prevention.

Sokoloff, N. J., \& Dupont, I. (2005). Domestic violence at the intersections of race, class, and gender: Challenges and contributions to understanding violence against marginalized women in diverse communities. Violence Against Women, 11(1), 38-64. https://doi. org/10.1177/1077801204271476

Starr, R. W. (2018). Moving from the mainstream to the margins: Lessons in culture and power. Journal of Family Violence, 33(8), 551-557. https://doi.org/10.1007/s10896-018-9984-1

Starr R.W. (2020). Transformational collaboration. National Resource Center for reaching victims and Caminar Latino. Retrieved from: TransformationalCollaboration-100920-Rev6.pdf (reachingvictims.org). Accessed 9/3/2021.

Stockman, J. K., Hayashi, H., \& Campbell, J. C. (2015). Intimate partner violence and its health impact on ethnic minority women. Journal of Women's Health, 24(1), 62-79. https://doi.org/10.1089/ jwh.2014.4879

Stogner, J., Miller, B. L., \& McLean, K. (2020). Police stress, mental health, and resiliency during the COVID-19 pandemic. American Journal of Criminal Justice, 45(4), 718-730.

Sullivan, C. M., \& Goodman, L. A. (2019). Advocacy with survivors of intimate partner violence: What it is, what it Isn't, and why It's critically important. Violence Against Women, 25(16), 2007-2023. https://doi.org/10.1177/1077801219875826

Tai, D., Shah, A., Doubeni, C. A., Sia, I. G., \& Wieland, M. L. (2021). The disproportionate impact of COVID-19 on racial and ethnic minorities in the United States. Clinical Infectious Diseases : An Official Publication of the Infectious Diseases Society of America, 72(4), 703-706. https://doi.org/10.1093/cid/ciaa815

Tahirih Justice Center. (2020, March 23). Analysis: The impact of covid-19 on immigrant survivors of gender-based violence " Tahirih Justice Center. Retrieved March 17, 2021, from https:// www.tahirih.org/pubs/the-impact-of-covid-19-on-immigrantsurvivors-of-gender-based-violence/

U.S. Department of Justice (USDOJ) Office on Violence Against Women. (2017, January). Reflections from the Field on Victim/ Survivor Advocacy. https://www.justice.gov/ovw/page/file/ 936746/downloadAccessed 7/1/2021

Van Der Feltz-Cornelis, C. M., Varley, D., Allgar, V. L., \& de Beurs, E. (2020). Workplace stress, Presenteeism, absenteeism, and resilience Amongst University staff and students in the COVID-19 
lockdown. Frontiers in Psychiatry, 11, 588803. https://doi.org/ $10.3389 /$ fpsyt.2020.588803

van Dernoon Lipinsky, L., \& Burk, C. (2009). Trauma stewardship: An everyday guide to caring for self while caring for others. BerrettKoehler Publishers.

World Health Organization (2021) Devastatingly pervasive: 1 in 3 women globally experience violence Accessed at: https://www. who.int/news/item/09-03-2021-devastatingly-pervasive-1-in-3women-globally-experience-violenceAccessed 7/1/2021

Wood, L. (2017). "I look across from me and I see me" survivors as advocates in intimate partner violence agencies. Violence Against Women, 23(3), 309-329.

Wood, L., Wachter, K., Rhodes, D., \& Wang, A. (2019). Turnover intention and job satisfaction among the intimate partner violence and sexual assault workforce. Violence and Victims, 34(4), 678700. https://doi.org/10.1891/0886-6708.VV-D-18-00134

Wood, L., Schrag, R. V., Baumler, E., Hairston, D., Guillot-Wright, S., Torres, E., \& Temple, J. R. (2020). On the front lines of the
COVID-19 pandemic: Occupational experiences of the intimate partner violence and sexual assault workforce. Journal of interpersonal violence, 886260520983304 . Advance online publication. https://doi.org/10.1177/0886260520983304

Wood, L., Cook Heffron, L., Voyles, M., \& Kulkarni, S. (2020b). Playing by the rules: Agency policy and procedure in service experience of IPV survivors. Journal of Interpersonal Violence, 35(2122), 4640-4665. https://doi.org/10.1177/0886260517716945

Woodlock, D. (2017). The abuse of technology in domestic violence and stalking. Violence Against Women, 23(5), 584-602.

Publisher's Note Springer Nature remains neutral with regard to jurisdictional claims in published maps and institutional affiliations.

\section{Authors and Affiliations}

\section{Rebecca Garcia ${ }^{1}$. Cynterria Henderson ${ }^{2} \cdot$ Kimberly Randel $^{3,4,5}$. Andrés Villaveces ${ }^{6}$ - Abbey Katz ${ }^{7}$. Fatimah Abioye ${ }^{8}$. Sarah DeGue ${ }^{6} \cdot$ Kelley Premo $^{2} \cdot$ Summer Miller-Wallish ${ }^{2} \cdot$ Judy C. Chang $^{9} \cdot$ Elizabeth Miller $^{2} \cdot$ Maya I. Ragavan $^{10,11}$ (1)}

Rebecca Garcia

rebeccakelley1110@gmail.com

Cynterria Henderson

cynterria.henderson@chp.edu

Kimberly Randell

karandell@cmh.edu

Andrés Villaveces

nnp4@cdc.gov

Abbey Katz

aKatz@futureswithoutviolence.org

Fatimah Abioye

abioye@ aap.org

Sarah DeGue

hci2@cdc.gov

Kelley Premo

Kelley.premo@chp.edu

Summer Miller-Wallfish

summer.millerwalfish@chp.edu

Judy C. Chang

changjc@upmc.edu

Elizabeth Miller

elizabeth.miller@chp.edu

1 Women's Center \& Shelter of Greater Pittsburgh, PO Box 9024, Pittsburgh, PA 15224, USA

2 Division of Adolescent and Young Adult Medicine, University of Pittsburgh, 120 Lytton Avenue, Pittsburgh, PA 15213, USA
3 Division of Pediatric Emergency Medicine, Children's Mercy Kansas City, 2401 Gillham Rd, Kansas City, MO 64110, USA

4 University of Kansas City-Missouri School of Medicine, Kansas City, MO, USA

5 University of Kansas School of Medicine, Kansas City, KS, USA

6 Division of Violence Prevention, National Center for Injury Prevention and Control, Centers for Disease Control and Prevention, 4770 Buford Highway., S106-10, Atlanta, GA 30341, USA

7 Futures Without Violence, 101 Montgomery Street, San Francisco, CA 94129, USA

8 Child Welfare, Trauma, and Resilience Initiatives, American Academy of Pediatrics, 345 Park Blvd, Itasca, IL 60413, USA

9 Department of Obstetrics, Gynecology \& Reproductive Sciences, and Internal Medicine, University of Pittsburgh, 300 Halket Street, Pittsburgh, PA 15213, USA

10 Division of General Academic Pediatrics, University of Pittsburgh, 3420 Fifth Avenue, Pittsburgh, PA 15213, USA

11 University of Pittsburgh, 3415 Fifth Avenue, Pittsburgh, PA 15213, USA 